\title{
Fast algorithm for estimation of the orientation term of a general quadrature transform with application to demodulation of an $n$-dimensional fringe pattern
}

\author{
Daniel Crespo, Juan Antonio Quiroga, and Jose Antonio Gomez-Pedrero
}

\begin{abstract}
The spatial orientation of fringes has been demonstrated to be a key point in reliable phase demodulation from a single $n$-dimensional fringe pattern, regardless of the frequency spectrum of the signal. Recent publications have shown a general method for determination of the orientation factor by use of a regularized phase-tracking (RPT) algorithm. We propose a generalization of a RPT algorithm for estimation of the spatial orientation in a general $n$-dimensional case. The proposed algorithm makes use of a simplified cost function that remains one dimensional regardless of the dimension of the problem. This makes the calculation faster than with a standard RPT algorithm, with which it is necessary to minimize an $n+1$-dimensional cost function for each point of the sample space. We have applied the method to the three-dimensional demodulation of a time-evolving fringe pattern, with good results. (C) 2004 Optical Society of America

OCIS codes: $\quad 100.2650,100.5070$.
\end{abstract}

\section{Theoretical Foundations}

A. Phase Demodulation with a Quadrature Operator and the Relevance of the Orientation Term

The irradiance distribution of a fringe pattern can be represented in a first approximation as an $n$ dimensional phase-modulated signal given by

$$
I(\mathbf{r})=b(\mathbf{r})+m(\mathbf{r}) \cos \phi(\mathbf{r}),
$$

were $I$ is the irradiance, $b$ is the background, $m$ is the modulation, $\phi$ is the modulating phase, and $\mathbf{r}=$ $\left(x_{1}, \ldots, x_{n}\right)$ denotes an $n$-dimensional position vector.

In most practical cases we can assume that the background is spatially smooth and that it can be removed with a high-pass filter. We denote by $I_{\mathrm{HP}}$

The authors are with the Departamento de Óptica, Facultad de Ciencias Físicas, Universidad Complutense, Ciudad Universitaria s/n 28040 Madrid, Spain. D. Crespo's e-mail address is daniel. crespo@indizen.com.

Received 8 July 2004; revised manuscript received 2 September 2004; accepted 8 September 2004.

0003-6935/04/336139-08\$15.00/0

(C) 2004 Optical Society of America the high-pass filtered, background-suppressed version of the irradiance distribution:

$$
I_{\mathrm{HP}}(\mathbf{r})=m(\mathbf{r}) \cos \phi(\mathbf{r}) .
$$

Usually the modulating phase is associated with the physical magnitude to be measured and the background and the modulation are associated with environmental conditions such as illumination setup and object reflectance. The process of phase demodulation consists in extracting the relevant information, $\phi(\mathbf{r})$, from an observed irradiance distribution of the form given by Eq. (2).

Phase demodulation from a single fringe pattern is an important task in areas such as the study of fast transient phenomena, in which only one fringe pattern can be acquired [two-dimensional (2D) case] or in which a temporal set can be captured but with unknown temporal behavior of the phase [threedimensional (3D) case].

In the general case of fringe patterns with closed fringes it is not possible to obtain good results with ordinary algorithms such as Fourier-transform and spatial phase sampling methods. ${ }^{1}$

One of the methods for solving this problem that was proposed recently is a general quadrature transform (GQT). ${ }^{2}$ The GQT is a quadrature operator, 
$Q_{n}\{\}$, which means that it is an operator that transforms a given fringe pattern into its quadrature term, $Q_{n}\left\{I_{\mathrm{HP}}(\mathbf{r})\right\}=-m(\mathbf{r}) \sin \phi(\mathbf{r})$. With this quadrature signal one can easily determine the modulo $2 \pi$ wrapped phase, $W\{\phi(\mathbf{r})\}$, over the whole region of interest by a simple arctangent calculation.

As was shown in Ref. 2, the GQT is given by

$$
Q_{n}\left\{I_{\mathrm{HP}}(\mathbf{r})\right\}=\frac{\nabla \phi(\mathbf{r})}{|\nabla \phi(\mathbf{r})|} \frac{\nabla I_{\mathrm{HP}}(\mathbf{r})}{|\nabla \phi(\mathbf{r})|}=\mathbf{n}_{\phi}(\mathbf{r}) \frac{\nabla I_{\mathrm{HP}}(\mathbf{r})}{|\nabla \phi(\mathbf{r})|} .
$$

The first term of Eq. (3), $\mathbf{n}_{\phi}(\mathbf{r})=\nabla \phi(\mathbf{r}) /|\nabla \phi(\mathbf{r})|$, is a unit vector normal to the corresponding isophasics, which points in the direction of a fringe orientation term denominated $\nabla \phi(\mathbf{r})$. The second term is an isotropic $n$-dimensional generalization of the onedimensional Hilbert transform, $\mathbf{H}_{n}\left\{I_{\mathrm{HP}}\right\}=\nabla I_{\mathrm{HP}} /$ $|\nabla \phi|$.

The term $\mathbf{H}_{n}\left\{I_{\mathrm{HP}}\right\}$ can be estimated as a linear operator, regardless of the dimension of the problem. ${ }^{2}$ But, for fringe patterns with closed fringes, the calculation of the orientation term $\mathbf{n}_{\phi}$ is a nonlinear problem.

To obtain the orientation term we have access only to the fringe pattern irradiance and its gradient. If modulation $m(\mathbf{r})$ and background $b(\mathbf{r})$ are spatially smooth, we can approximate $\nabla I \approx-m \sin \phi \nabla \phi$, and then the unitary irradiance gradient can be written as

$$
\tilde{\mathbf{n}}_{\phi}(\mathbf{r})=\frac{\nabla I}{|\nabla I|}=\frac{-\sin \phi}{|\sin \phi|} \frac{\nabla \phi(\mathbf{r})}{|\nabla \phi(\mathbf{r})|}=-\operatorname{sign}(\sin \phi) \mathbf{n}_{\phi}(\mathbf{r}) .
$$

Equation (4) states that we can compute orientation term $\mathbf{n}_{\phi}$ from the irradiance gradient if we can estimate the fringe pattern quadrature sign (QS), given by

$$
\mathrm{QS}\{I\}=-\operatorname{sign}(\sin \phi) \text {. }
$$

As is shown in Ref. 3, the calculation of the QS in a general $n$-dimensional case is possible by unwrapping of a generalization of the 2D fringe orientation angles. For the sake of clarity we include here a brief description of this method. In effect, if we define the angles

$$
\begin{aligned}
\beta_{k}{ }^{(\pi)} & =\arctan \left(\frac{-\partial I / \partial x_{k+1}}{\partial I / \partial x_{k}}\right), \\
\beta_{k}{ }^{(2 \pi)} & =\arctan \left(\frac{-\partial \phi / \partial x_{k+1}}{\partial \phi / \partial x_{k}}\right),
\end{aligned}
$$

where $k$ can take any value from 1 to the number of dimensions $n$, the relationship between $\cos \beta_{k}{ }^{(\pi)}$ and $\cos \beta_{k}^{(2 \pi)}$ is

$$
\cos \beta_{k}{ }^{(\pi)}=-\operatorname{sign}(\sin \phi) \cos \beta_{k}^{(2 \pi)}=\mathrm{QS}\{I\} \cos \beta_{k}{ }^{(2 \pi)} .
$$

From Eq. (6) it follows that the problem of calculating the QS can be reduced to the problem of calculating $\beta_{k}{ }^{(2 \pi)}$, which cannot be directly obtained from the irradiance. $\beta_{k}{ }^{(\pi)}$, however, is a direct measurement but because of the sign flips of the irradiance gradient is defined only modulo $\pi{ }^{4}$ However, as was shown in Ref. 5 , it is possible to recover $\beta_{k}{ }^{(2 \pi)}$ by unwrapping $W\left\{2 \beta_{k}{ }^{(\pi)}\right\}$, where $W\{\}$ denotes the wrapping operator. To calculate the QS, one needs to perform this unwrapping process only for one arbitrary choice of axis $k$, let us say $k_{0}$. To ease the following discussion, we refer to $\beta_{k_{0}}{ }^{(\pi)}$ simply as $\beta$.

So the problem of demodulating an $n$-dimensional fringe pattern is reduced to the use of a linear operator $\mathbf{H}_{n}\left\{I_{\mathrm{HP}}\right\}$ plus the problem of unwrapping the distribution $W\{2 \beta\}$, where $\beta$ can be calculated directly from the gradients of the observed irradiance distribution. Because $\beta$ is an angular magnitude, for patterns with closed fringes the distribution $2 \beta$ will be piecewise continuous, with discontinuities of $\pm 4 \pi$ for paths that enclose a fringe center and pairs of poles of the same sign at the origin of the closed fringes. ${ }^{6}$ So, in general, the process of unwrapping $W\{2 \beta\}$ will be path dependent, ${ }^{5}$ and in consequence it will not be possible to use standard phase-unwrapping algorithms.

\section{B. Unwrapping Based on a Regularized Phase-Tracking Orientation}

The problem of unwrapping $W\{2 \beta\}$ has been addressed by Quiroga et al. for the $2 \mathrm{D}$ case, ${ }^{5}$ where there is only one possible choice of $k$ (let us say $k=1$ ) and computation of the modulo $2 \pi$ fringe orientation angle and the QS is equivalent. Following Ref. 5, one can unwrap $W\{2 \beta\}$ by using a regularized phasetracking (RPT) procedure, ${ }^{7}$ computing two signals in quadrature given by

$$
\begin{aligned}
& f_{C}(r)=\cos (W\{2 \beta\}), \\
& f_{S}(r)=\sin (W\{2 \beta\})
\end{aligned}
$$

and demodulating the phase of these signals by minimizing at each point the cost function given by

$$
\begin{aligned}
U_{r}\left(\theta, \omega_{x}, \omega_{y}\right)= & \sum_{\xi \eta \in N \cap L}\left|f_{C}(\xi, \eta)-\cos p(x, y, \xi, \eta)\right|^{2} \\
& +\left|f_{S}(\xi, \eta)-\sin p(x, y, \xi, \eta)\right|^{2}+\cdots \\
& +\mu \mid W_{4 \pi}\{\theta(\xi, \eta) \\
& -p(x, y, \xi, \eta)\}\left.\right|^{2} m(\xi, \eta),
\end{aligned}
$$

where

$$
\begin{aligned}
p(x, y, \xi, \eta)= & \theta(x, y)+\omega_{x}(x, y)(x-\xi) \\
& +\omega_{y}(x, y)(y-\eta),
\end{aligned}
$$

where $L$ is the region with valid data and $N$ is a neighborhood region about point $\mathbf{r}$ where the phase is being calculated. Function $m(\xi, \eta)$ is an indicator that equals 1 if the point has already been estimated and equals 0 otherwise, and finally $\mu$ is the so-called regularization parameter.

The RPT process finds, for each successive point $\mathbf{r}$, the values of $\theta, \omega_{x}$, and $\omega_{y}$ that minimize the cost 


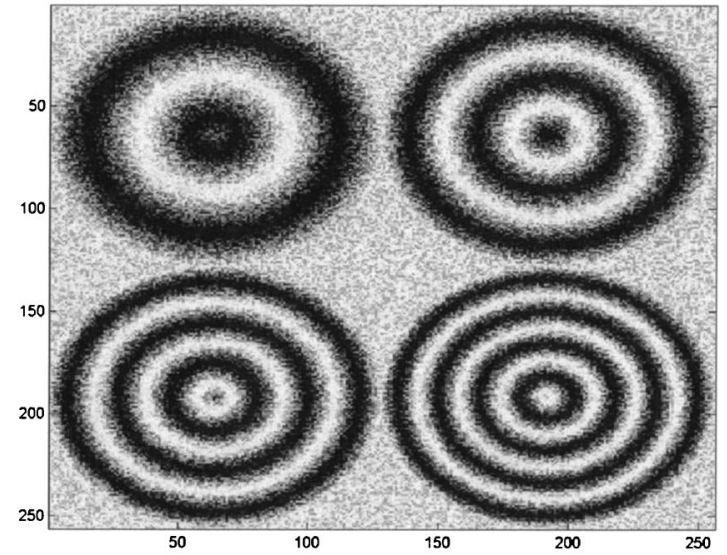

(a)

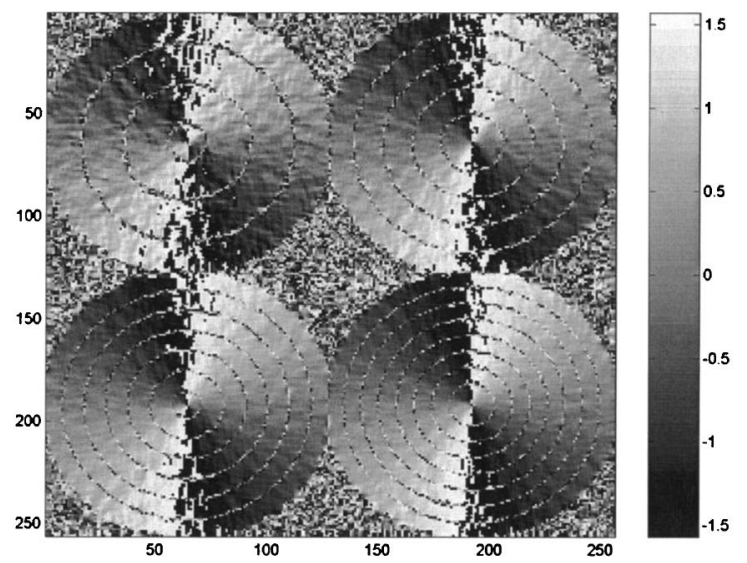

(b)

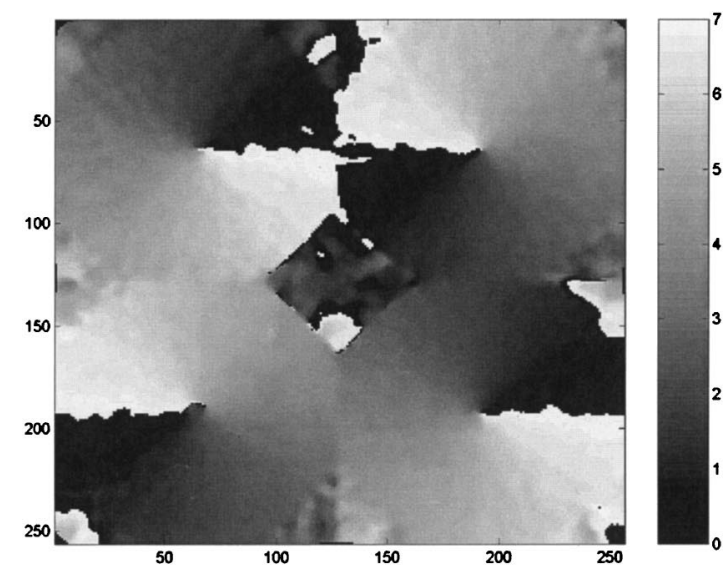

(c)

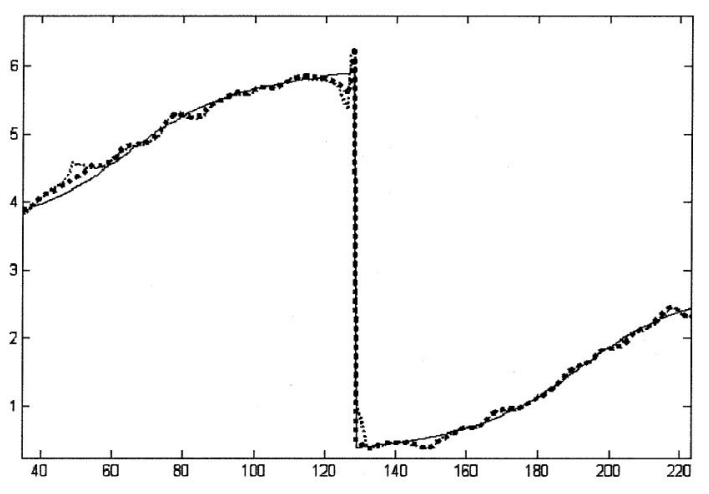

(d)

Fig. 1. (a) Simulated fringe pattern with four isolated circular regions and random noise. (b) $\beta_{1}{ }^{\pi}$ calculated from Eq. (6). (c) Unwrapped orientation map obtained with the algorithm presented in this paper. (d) Comparison of the results obtained with a standard RPT algorithm (dashed curve), our optimized algorithm (dotted curve), and the theoretical value for the orientation (continuous curve).

function. The value of $\theta$ obtained at each point $\mathbf{r}$ will correspond to the unwrapped value of $2 \beta$.

The first two terms in Eq. (8) correspond to the so-called fidelity term of the RPT; they try to adjust the observations $f_{C}$ and $f_{S}$ to sinusoidal functions modulated by a plane. They correspond to the assumptions that $f_{C}$ and $f_{S}$ are locally monochromatic and therefore that $\omega_{x}$ and $\omega_{y}$ can be interpreted as the local frequencies of the recovered solution.

The last term in Eq. (8) is the so-called regularization term, and it enforces smoothness and continuity of the recovered solution. In this case we are unwrapping distribution $2 \beta$, which we know has to be continuous modulo $4 \pi,{ }^{1}$ aside from possible isolated discontinuities in the center of closed fringes. The regularization term forces the continuity of the solution modulo $4 \pi$, and this is denoted in Eq. (8) by the term with $W_{4 \pi}\{\}$. This is the main difference between the normal RPT technique for phase demodulation and the RPT applied to the calculation of orientation terms.

The RPT algorithm is implemented as follows: Starting at a given point $\mathbf{r}_{0}$, the cost function is minimized for that point and initial values are obtained for $\theta, \omega_{x}$, and $\omega_{y}$. The value of $m\left(\mathbf{r}_{0}\right)$ is up- dated to 1 , and then we proceed to do the same calculation for the neighboring points of $\mathbf{r}_{0}$. From each point the algorithm moves to the next noncalculated neighbor, until the whole region of interest has been calculated.

The strategy for choosing the order of the neighbors, and therefore the order in which the region of interest will be explored to yield the solution, is important and is the determinant of the robustness of the RPT method. For the demodulation of $2 \beta$ an isophasic scanning 5 strategy is used, which means that the solution is obtained following the directions along which the phase remains constant. This is the orthogonal direction to $\omega=\nabla \phi$.

\section{Proposed Method}

We show a generalization to the $n$-dimensional case of the procedure shown above, and we introduce an approximation that will make the RPT calculation faster, especially as the number of dimensions $n$ increases.

For the $n$-dimensional case it is straightforward that one can use the same RPT method described 
above for the $2 \mathrm{D}$ case to unwrap $W\left\{2 \beta_{k}{ }^{(\pi)}\right\}$, where $1 \leq$ $k \leq n$. In this case $U_{\mathbf{r}}$ becomes just

$$
\begin{aligned}
U_{\mathbf{r}}(\theta, \boldsymbol{\omega})= & \sum_{\xi \in N \cap L}\left|f_{C}(\boldsymbol{\rho})-\cos p(\mathbf{r}, \boldsymbol{\rho})\right|^{2}+\mid f_{S}(\boldsymbol{\rho}) \\
& -\left.\sin p(\mathbf{r}, \boldsymbol{\rho})\right|^{2}+\cdots+\mu \mid W_{4 \pi}\{\theta(\boldsymbol{\rho}) \\
& -p(\mathbf{r}, \boldsymbol{\rho})\}\left.\right|^{2} m(\mathbf{\rho})
\end{aligned}
$$

where $\mathbf{r}, \boldsymbol{\rho}$, and $\boldsymbol{\omega}$ are $n$-dimensional vectors, $p(\mathbf{r}, \boldsymbol{\rho})=$ $\theta(\mathbf{r})+\boldsymbol{\omega}(\mathbf{r})(\mathbf{r}-\boldsymbol{\rho})$, and $U_{\mathbf{r}}$ is an $n+1$-dimensional function that has to be minimized at each point of the $n$-dimensional region of interest. Typical minimization algorithms are $O\left(n^{2}\right)$, so the time taken to calculate the global RPT process will be $O\left[(n+1)^{2}\right] M$, where $M$ is the total number of points (one minimization in $n+1$ dimensions for each point of the $n$-dimensional region of interest).

Using the RPT technique as described above, the algorithm will find the best locally monochromatic function that provides a smooth and continuous solution for $2 \beta$. In the process, the algorithm adjusts at each point $\mathbf{r}$ the value of the local phase $\theta$, and also the value of the local spatial frequencies, the $n$ components of the $\omega$ vector.

But $2 \beta$ is usually a smooth, slowly varying function, and we can assume that spatial frequencies $\boldsymbol{\omega}$ that are output from the RPT will not be much different from the estimation $\hat{\boldsymbol{\omega}}$ that can be obtained directly from the observed $W\{2 \beta\}$; that is, we can use the next approximation:

$$
\boldsymbol{\omega}(\mathbf{r}) \approx \hat{\boldsymbol{\omega}}(\mathbf{r})=\nabla 2 \beta(\mathbf{r})=W\{\nabla W\{2 \beta(\mathbf{r})\}\} .
$$

Relation (11) will hold true for those points where $2 \beta$ is continuous and is well sampled. The value of $\hat{\boldsymbol{\omega}}$ given relation (11) is also a good approximation for those points at which $2 \beta$ presents a $4 \pi$ discontinuity because as follows from Eqs. (9) and (10), a jump in $2 \beta$ of $4 \pi$ does not modify the value of $U_{r}$.

So, using the approximation given by relation (11), we can simplify the minimization: we have only to look for the value of $\theta$ that minimizes $U_{\mathbf{r}}$ at each point r. The cost function now becomes one dimensional, regardless of the dimension $n$ of the problem:

$$
\begin{aligned}
U_{\mathbf{r}}(\theta)= & \sum_{\xi \in N \cap L}\left|f_{C}(\boldsymbol{\rho})-\cos \hat{p}(\mathbf{r}, \boldsymbol{\rho})\right|^{2}+\mid f_{S}(\boldsymbol{\rho}) \\
& -\left.\sin \hat{p}(\mathbf{r}, \boldsymbol{\rho})\right|^{2}+\cdots+\mu \mid W_{4 \pi}\{\theta(\boldsymbol{\rho}) \\
& -\hat{p}(\mathbf{r}, \boldsymbol{\rho})\}\left.\right|^{2} m(\boldsymbol{\rho}),
\end{aligned}
$$

where $\hat{p}(\mathbf{r}, \boldsymbol{\rho})=\theta(\mathbf{r})+\hat{\boldsymbol{\omega}}(\mathbf{r})(\mathbf{r}-\boldsymbol{\rho})$. This means that the time consumed by the RPT algorithm now becomes proportional only to $M$ and that for $n>1$ the time consumed should be significantly smaller than in the original RPT technique.

With this proposed method we are requiring that the local spatial frequencies of the unwrapped solution be those given by relation (11). This means that the values of $\boldsymbol{\omega}$ are being calculated with only next neighbors for each point taken into account. This

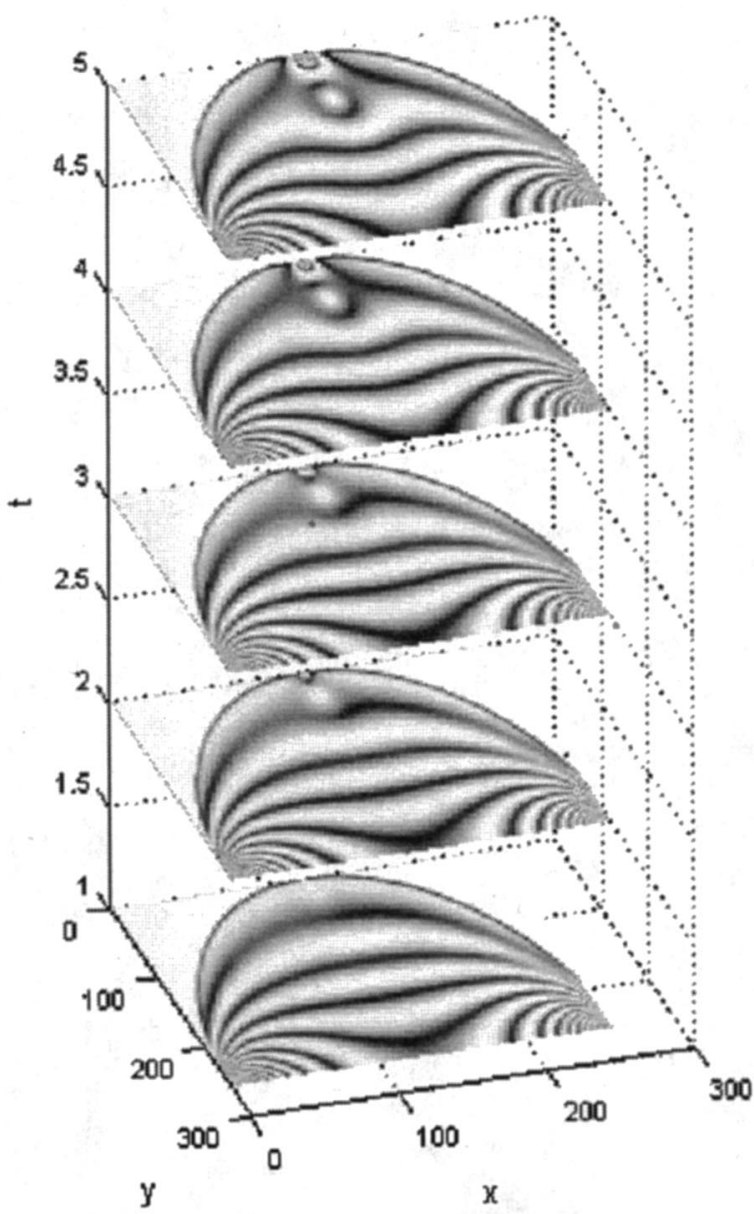

Fig. 2. Consecutive snapshots, obtained with a polariscope, of the isochromatics on a disk of a photoelastic material when an increase of the mechanical load is progressively applied on its upper side.

implies that the size of neighborhood region $N$ in Eq. (12) must be kept small to produce consistent results in the minimization process. In the new algorithm a typical size of $N$ would be $3^{n}$. Indirectly, this size results in an extra time optimization, as the neighborhood region for a typical RPT problem in which both $\theta$ and $\omega$ have to be estimated at each point is usually larger, typically $7^{n}-11^{n}$.

In conclusion, the proposed algorithm is faster than the traditional RPT and scales better with the dimension of the problem while it retains the advantages that make RPT so robust, such as adaptative bandpass filtering, adaptive masking, ${ }^{8}$ and use of quality map-guided scanning and isophasic scanning. ${ }^{2,7}$

\section{Results}

First we show an example of the 2D case and compare the results obtained with the algorithm presented in this paper with those obtained with the standard RPT algorithm. Figure 1(a) shows a $256 \times 256$ computer-generated fringe pattern containing four separate circular regions. Each region contains circular fringes with different spatial 


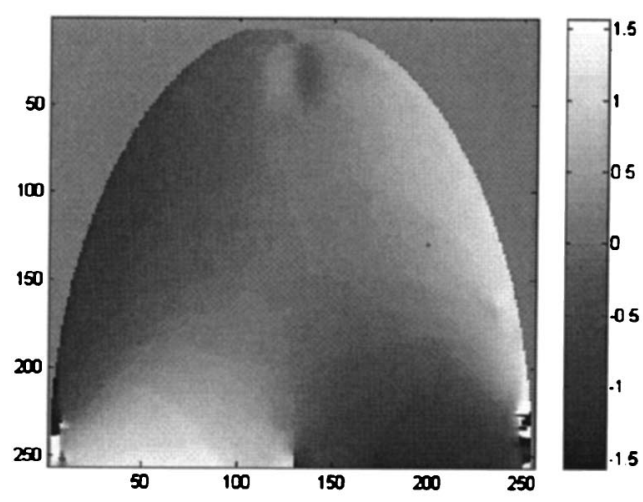

(a)

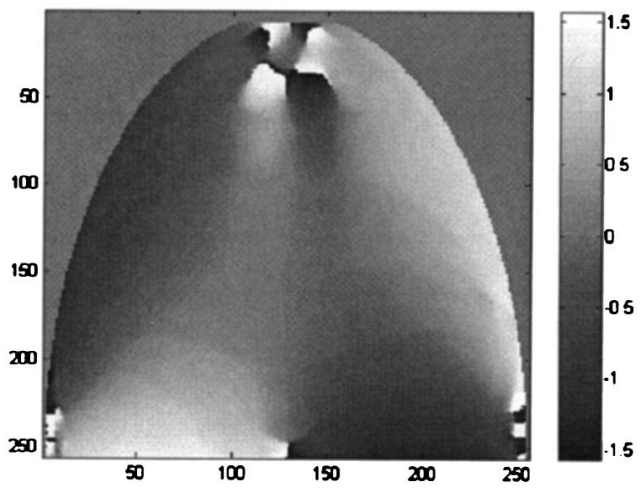

(c)

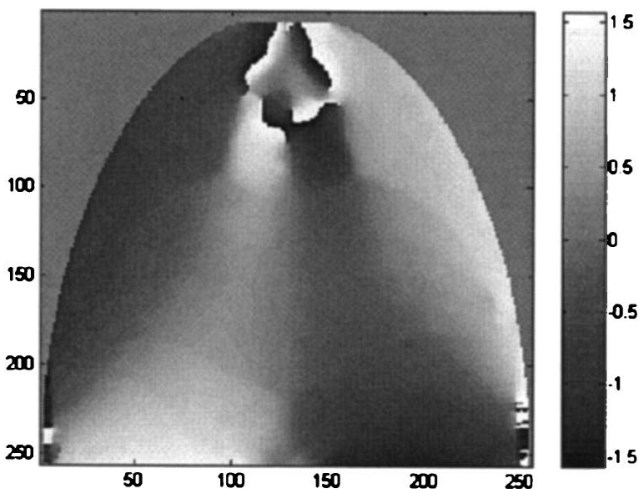

(e)

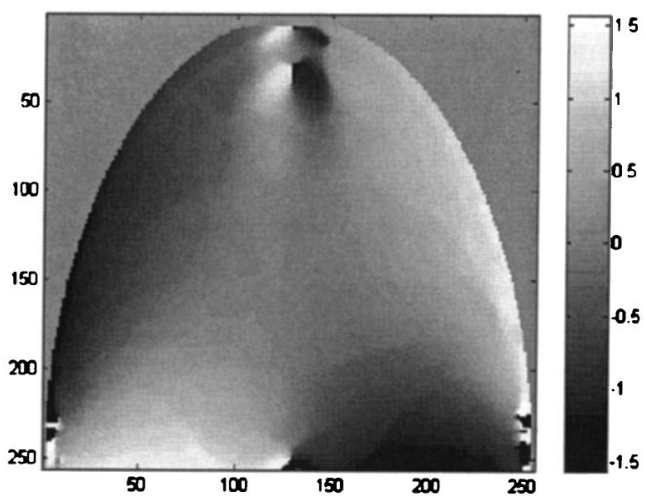

(b)

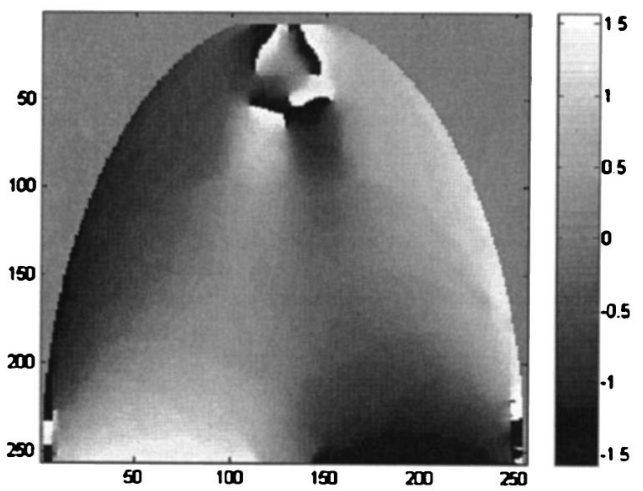

(d)

Fig. 3. $\beta_{1}{ }^{\pi}$ for each of the consecutive snapshots (or planes along the $t$ axis).

frequencies. A random noise factor of 0.4 has been added to the figure. Figure $1(\mathrm{~b})$ shows $\beta_{1}{ }^{\pi}$ obtained from Fig. 1(a) by use of Eq. (6). Figure 1(c) shows the modulo $2 \pi$ orientation map obtained with the algorithm presented in this paper. Figure 1(d) shows a comparison, along row 90 of the test image, of the results obtained with a standard RPT algorithm (dashed curve), the new algorithm (dotted curve), and the theoretical value of the modulo $2 \pi$ orientation (continuous curve). For both calculations, by the standard RPT and by the optimized algorithm, we used values of $\mu=6$ and a neighbor- 


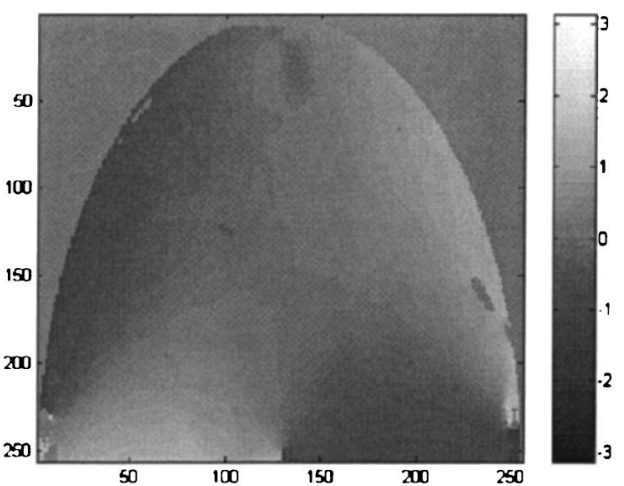

(a)

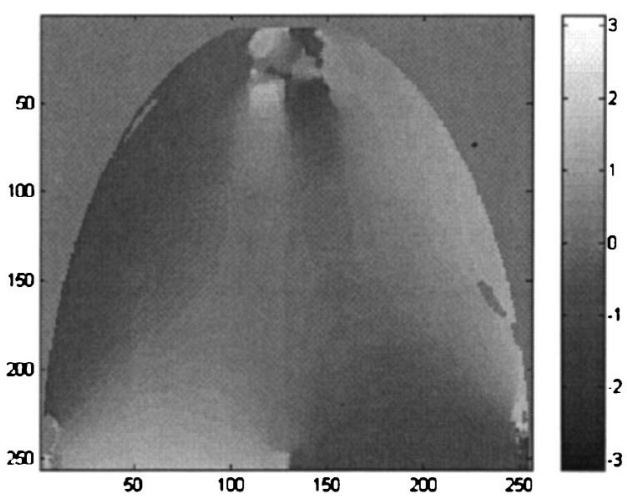

(c)

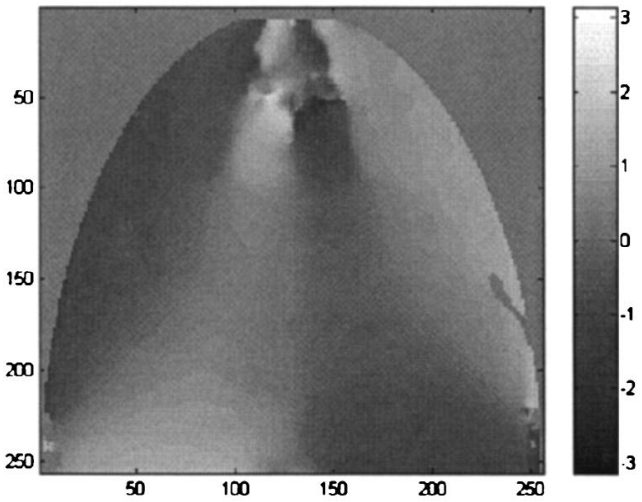

(e)

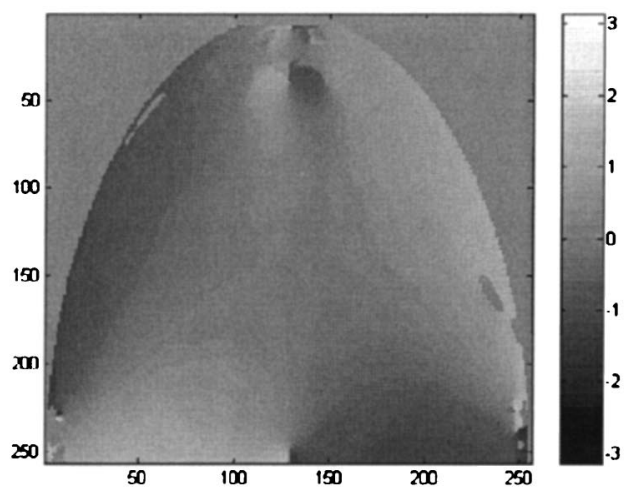

(b)

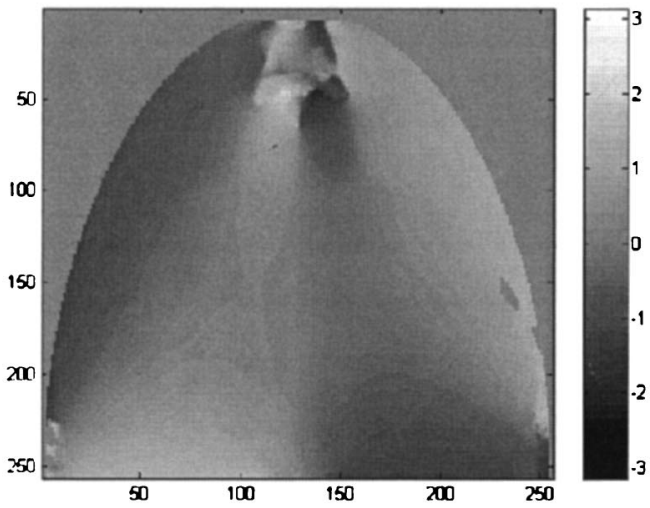

(d) 


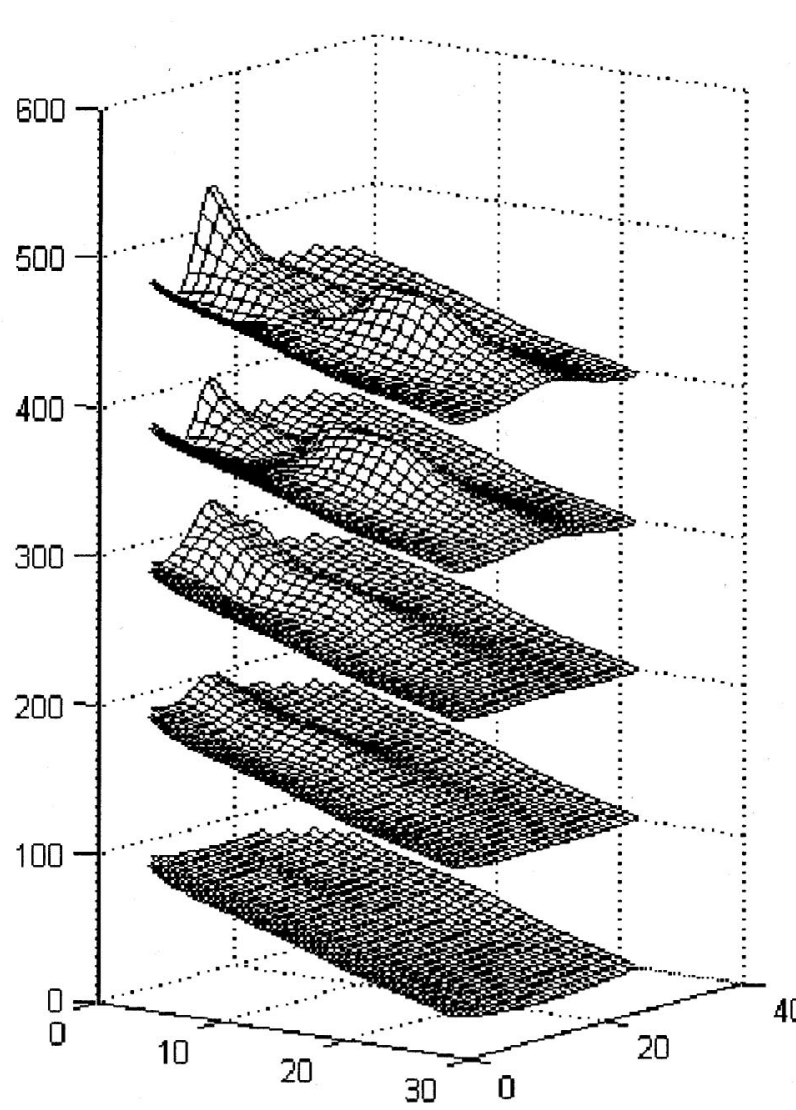

(a)

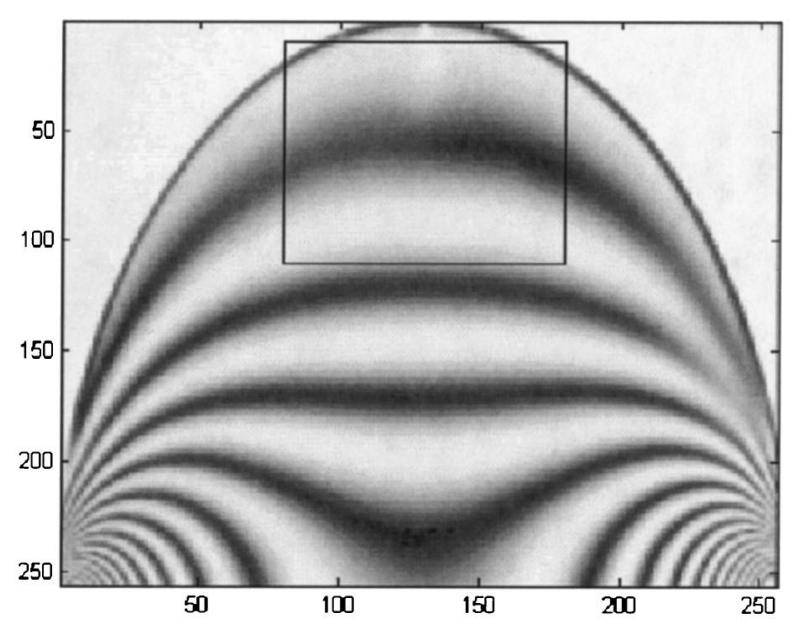

(b)

Fig. 5. (a) Final recovered values of the phase for each consecutive snapshot, obtained with the GQT algorithm. (b) Values of the phase shown in (a) that correspond to the region marked by the black rectangle. This is the region where the change in phase between successive snapshots is greatest.

applied our algorithm to a set of fringe patterns that evolves in time. Figure 2 shows the circular bright field isochromatic fringe patterns of a stress-frozen diametrically compressed disk as a load on the upper side of the disk is increased. This case can be treated as a $3 \mathrm{D}$ fringe pattern with $\mathbf{r}=(x, y, t)$. In Fig. 3 we show $\beta_{1}{ }^{(\pi)}=\arctan (-\partial I / \partial y / \partial I / \partial x)$ for each of the consecutive time instants (or planes along the $t$ axis). In Fig. 4 we show the results obtained for the unwrapping of $W\left\{2 \beta_{1}{ }^{\pi}\right\}$ by using the algorithm presented in this paper. On a Pentium $42.1-\mathrm{GHz}$ word processor the time taken for the whole calculation was $92.6 \mathrm{~s}$. The same calculation by a standard RPT method extended to the $3 \mathrm{D}$ case took $310.4 \mathrm{~s}$. As was expected, the time difference between the two methods increased as the dimension of the problem increased.

In Fig. 5(a) we show the final phase distribution $\phi(\mathbf{r})$ that would be obtained by application of the GQT given by Eq. (3) by use of the 3D generalization of the one-dimensional Hilbert transform as shown in Ref. 5 and of the orientation term obtained with the present algorithm. The values shown in Fig. 5(a) correspond to the region outlined by the black rectangle in Fig. $5(\mathrm{~b})$; this is the region where the changes between snapshots of the applied load are greater and therefore where the temporal evolution of the underlying phase is seen more clearly.

\section{Conclusions}

The general quadrature transform is a method that was recently presented by Servin et al..$^{2}$ and can be used for the demodulation of phase maps from a single $n$-dimensional fringe pattern. We have presented a new algorithm, based on a generalization to $n$ dimensions of the RPT technique, ${ }^{3,5}$ for calculating the orientation term of the GQT in a general $n$-dimensional case. The proposed algorithm introduces an approximation, suitable for the calculation of orientation terms, that makes the algorithm faster than the standard RPT while it retains the advantages (adaptive bandpass filtering, use of quality maps, isophasic scanning) that make it so robust. The proposed method can be used also as a phaseunwrapping technique for a general phase map without the special characteristics of the orientation fields.

We are grateful for financial support of this research by project DPI2002-02104 of the Ministerio de Ciencia y Tecnología of Spain and by Airbus France and NDT Expert under the contract "Development of new methods for the demodulation of moiré fringe patterns."

\section{References}

1. T. Kreis, Holographic Interferometry (Akademie Verlag, Berlin, 1996).

2. M. Servin, J. A. Quiroga, and J. L. Marroquin, "A general $n$-dimensional quadrature transform and its application to interferogram demodulation," J. Opt. Soc. Am. A 20, 925-934 (2003).

3. J. A. Quiroga, M. Servin, J. L. Marroquin, and D. Crespo, "Estimation of the orientation term of the general quadrature transform from a single $n$-dimensional fringe pattern," submitted to J. Opt. Soc. Am. A.

4. X. Zhou, J. P. Baird, and J. F. Arnold, "Fringe-orientation estimation by use of a Gaussian gradient-filter and neighboringdirection averaging," Appl. Opt. 38, 795-804 (1999).

5. J. A. Quiroga, M. Servin, and F. Cuevas, "Modulo $2 \pi$ fringe- 
orientation angle estimation by phase unwrapping with a regularized phase tracking algorithm," J. Opt. Soc. Am. A 19, 1524-1531 (2002).

6. D. Ghiglia and M. D. Pritt, Two-Dimensional Phase Unwrapping (Wiley, New York, 1998).

7. M. Servin, F. J. Cuevas, D. Malacara, J. L. Marroquin, and R. Rodriguez-Vera, "Phase unwrapping through demodulation by use of the regularized phase-tracking technique," Appl. Opt. 35, 2192-2198 (1996).

8. J. A. Quiroga, M. Servin, and J. L. Marroquín, "Robust demodulation of isochromatics from a single tricolour image using an adaptive regularised phase tracking technique," presented at the international conference, PhotoMechanique 2001, presented at Poitiers, France, 24-26 April, 2001. 